\title{
The Association of Clinical Pathologists: 82nd General Meeting
}

The 82nd general meeting of the Association of Clinical Pathologists was held in Harrogate on 17, 18, and 19 April 1969. The programme included a symposium on 'The small intestine' at which invited speakers read the papers. This symposium will be published in the Journal later in the year. The second symposium was concerned with 'Radioisotopes in the pathology laboratory'. There was also a forum on the Zuckerman Report at which the opening speakers were J. L. Stafford and A. G. Marshall with the Editor of the Journal in the Chair. Abstracts of some of the remaining papers follow.

CARCINOMA IN SITU AND EARLY INFILTRATING CARCINOMA OF THE BRONCHUS

M. K. MASON (St James's Hospital, Leeds) A series of patients with a carcinoma in situ or early infiltrating carcinoma of the bronchus is described. Such lesions have been found in $2 \%$ of patients undergoing pneumonectomy or lobectomy for bronchial carcinoma. The carcinomas are all squamous in type and present in two ways. First, they may form an intrabronchial papillary mass, producing obstruction and gross radiological abnormalities. Secondly, there may be change to carcinoma in situ without the formation of a mass, so that $x$-ray changes are absent. Diagnosis of carcinoma in the first group is straightforward, but it is difficult to recognize that the lesion is an early one until after its removal. Preoperative diagnosis and localization in the second group is often very difficult and careful investigation is required. Carcinoma in situ may be suspected on clinical grounds in these patients as they often present with frequent small haemoptyses.

When a carcinoma in situ is found it is considered that it should be removed by as conservative a procedure as its site permits. Following excision, a good prognosis for the tumour can be expected. The significance of these lesions is discussed and it is suggested that they are frequently multifocal or potentially multifocal in origin. A prolonged follow up is required as there is an increased risk of a further carcinoma subsequently developing.

\section{ASBESTOS LEVELS IN HUMAN LUNGS}

C. Gold (M.R.C. Pneumoconiosis Research Unit, Penarth, Glamorgan) With the development of the KOH extraction method for the detection and quantitation of asbestos in lung tissue it has now become possible to evaluate the pathological changes observed in terms of the amount of asbestos present.

Over a period of three and a half years (1965 to 1969) a series of 620 extracts was examined from lung biopsies, lobectomies, pneumonectomies, and postmortem lungs. Three hundred and thirty-six were positive for asbestos. Two hundred and ninety-two of these were quantitated and related to the presence or absence of asbestos lung disease.

The values obtained ranged from nil to $13,000,000$ asbestos forms per gram of dried lung tissue and fitted fairly well into four main groups depending on whether there was no disease, mild, moderate, or severe asbestosis present.

The known association of asbestos and malignancy was confirmed but the pattern was less clear cut. All the extracts from the positive tumour cases gave abnormal results. Lung values varied considerably in pleural and peritoneal mesotheliomas and tumour extract levels were low. Bronchial carcinomas gave variably raised lung counts too but there was a difference in that the tumour tissue gave relatively high counts also.

In view of the widespread use of asbestos, the increasing number of reports showing the presence of asbestos in 20 to $60 \%$ of routine necropsies, and the known industrial and occupational risks, it would seem important to define significant disease levels. These could be used to control community and individual exposure risks while regulated utilization of a valuable mineral like asbestos continues.

\section{'APPARENTLY BARREN REGIONS' OF THE TEMPORAL BONE}

I. WRIGHT (Manchester) Many unsolved and interesting problems remain in the pathology of the petrous temporal bone. Too often these remain unexamined histologically because the general pathologist remains unaware of the paucity of material already available for study in certain diseases. A list of these was given, and removal of the temporal bone at necropsy was described.

\section{THE SUBSTANTIA NIGRA IN PARKINSONISM}

L. WOLMAN AND S. ROY (Department of Neuropathology, Royal Infirmary, Sheffield) A comparison of the changes in substantia nigra in three cases of idiopathic Parkinsonism seen by light and electron microscopy has been made. Characteristically there was a loss of pigmentation due to reduction in number of pigmented nerve cells, with residues of melanin lying free in the tissue or in histiocytes 
in perivascular spaces. Many remaining neurones contained one or more spherical concentric hyaline inclusion bodies of Lewy. Ultrastructurally these consisted of fibrillary and granular material with the fibrils more abundant at the periphery where they tended to be radially arranged. The granular component usually predominated in the centre where it formed a compact mass corresponding with the refractile and strongly acidophilic central core. Some Lewy bodies appeared larger and more homogeneous without any central core and these were composed entirely of uniformly dispersed fibrillary material. There was no evidence from electron microscopy that altered melanin granules entered into the composition of the inclusions, but occasionally degenerating organelles, particularly mitochondria, were seen in these structures, suggesting that they represented areas of cytoplasmic degeneration within the neurones.

Corpora amylacea, frequent in the substantia nigra of these cases, differed from the Lewy bodies both in situation and in ultrastructure, being composed of larger, coarser granules and fibrils partially enclosed by a limiting membrane. Although there was reduction in number of melanin granules in Parkinsonism no significant structural difference in the granules was found on electron microscopy in comparison with those from normal control material of similar age. Although chemically different, the ultrastructural similarity between melanin and lipofuscin was striking.

\section{A CLINICO-PATHOLOGICAL AND FAMILY STUDY OF POLYCYSTIC DISEASE OF THE KIDNEYS AND LIVER IN CHILDREN}

H. M. BLYTH AND B. G. OCKENDEN (M.R.C. Clinical Genetics Unit and Departmen: of Morbid Anatomy, Institute of Child Health, Guilford Street, London) The purpose of this study was to define those types of cystic disease of the kidneys occurring in children which carry a high risk of recurrence within the family. Difficulty had been experienced with genetic counselling for these conditions.

Index patients, having cystic malformation of the renal tubules and intrahepatic bile ducts (polycystic disease of the kidneys and liver), were ascertained ( $a$ ) from surgical and postmortem material examined at The Hospital for Sick Children and (b) from families referred to the Genetic Clinic at the hospital over the past 20 years. The latter were included only if the diagnosis could be confirmed histologically in at least one member of the family.

Twenty-nine cases in 24 families were studied in which the diagnosis was confirmed histologically; in three of these families a further six relatives have been included on clinical and/or radiological grounds.

Dominant inheritance is certain in one family and three others come into this 'adult' type on the histological criteria of focal cystic malformation of renal tubules and hepatic bile ducts.

In 20 families an autosomal recessive pattern of inheritance is indicated. In this 'childhood' type the cystic malformation affects all the intrahepatic bile ducts and the renal tubular lesions are uniformly dispersed throughout both kidneys. On clinico-pathological grounds the 25 cases in these families fell into four $\stackrel{2}{\stackrel{2}{2}}$ contiguous groups. The most easily recognized groups were those presenting earliest in the perinatal and neonatal periods, with bilateral large kidneys. A con- $\vec{P}$ trasting group presents in childhood with hepatomegaly을 and portal hypertension. Between these is a group $\bar{\sigma}$ presenting in infancy with hepatosplenomegaly and $\overline{\frac{\sigma}{\sigma}}$ developing renal insufficiency later. Within individual $\vec{\Phi}$ families the type of disorder breeds true, suggesting that each group represents a recessive condition, but that a $\infty$ different mutant gene is involved in each group.

This paper will be published in full.

\section{A CASE OF FATAL CLOSTIDIUM WELCHII TOXAEMIA DUE} TO WARD INFECTION

J. G. ALEXANDER (Royal Infirmary, Hull) A 14-day-old $\stackrel{\text { N }}{n}$ male baby had a small sacral meningocoele repaired in $\dot{\sigma}$ a neurosurgery theatre. There was no adrenalin in the local anaesthetic. He collapsed 20 hours later with meningitis due to Clostridium welchii (toxin type $\mathrm{A}$ 응 serologically untypable). Intravenous ampicillin and cloxacillin did not prevent death from acute haemolytic $\vec{c}$ anaemia 27 hours after operation. The organism was not present in his faeces. Histological examination of the wound edges showed morphological Clostridium welchii. From a large number of swabs from the theatre suite, the ward, and the portable incubator in which the baby $\square$ travelled from (but not to) the theatre, there were seven toxin type A serologically untypable strains isolated. A rabbit antiserum made against the baby's strain agglut- $\bar{O}$ inated two strains from the incubator and one from the

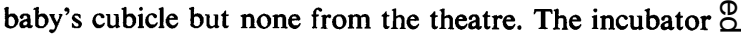
was not infected from the baby because of an imperme- $\overrightarrow{\vec{F}}$ able Nobecutane plastic film over the wound (causing $\frac{0}{3}$ tissue anaerobiosis). Previously, patients whose wounds yielded Clostridium welchii had not shown toxicity. Such a patient was the incubator's previous occupant and her Clostridium welchii had not been kept. This (or another ward strain) must have got on to the patient's skin in the $\bar{\sigma}$ ward. The bath used to bathe him preoperatively had 3 been previously wiped with Savlon. Three applications $\dot{\partial}$ of $0.5 \%$ chlorhexidine in $70 \%$ spirit to the operation area in the theatre did not prevent the infection. The local anaesthetic, Nobecutane spray, and dressings were sterile. Clostridium welchii strains were not found in the $D$ theatre air inlet ventilators or anywhere in the neurosurgery theatre.

\section{SIGNIFICANCE OF THE GIANT ORTHOCHROMATIC ERYTHROBLAST}

H. B. GOODALl (Royal Infirmary, Dundee) The giant orthochromatic erythroblast is a greatly enlarged poly-

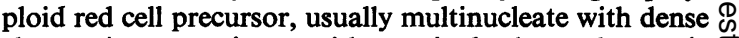
chromatin, sometimes with a single hyperchromatic? nuclear mass and with mature, well haemoglobinized cytoplasm. This characteristic morphological change may occur in the bone marrow in a wide range of clinical circumstances: as an inborn defect associated with ineffec- $\mathbb{D}$ tive erythropoiesis; as an accompaniment of neoplasia $\sigma$ of erythroblasts or lymphoreticular cells; in deficiency of 